\title{
Antibacterial peptides and the outer membranes of gram-negative bacilli
}

\begin{abstract}
Major problems are being experienced in human medicine from antibiotic resistant bacteria [1]. Furthermore, no new chemical class of antibiotics has been introduced into medicine in the past two decades [2]. Although many antibiotic structures have been subjected to intensive chemical manipulation to yield novel derivatives with extended activity spectra, resistance to such antibiotics has usually emerged quickly. An ideal antimicrobial agent, in the antibiotic resistance era, would be one that does not suffer intrinsic or induced resistance problems, or one that directly attacks a common resistance mechanism. Antibacterial peptides that represent a conserved theme in host antimicrobial defences throughout nature are of great interest in this regard and potentially provide the first novel class of antimicrobial agents since the introduction of nalidixic acid in 1970.
\end{abstract}

\section{Antibacterial peptides - nature's antibiotics}

Antibacterial peptides have been found in bacteria, fungi, plants, insects, amphibians, fish, crustaceans, birds, mammals and man [3]. These peptides are small (11-35 amino acids) and cationic by virtue of possessing several arginine and lysine residues. In species such as insects they (e.g., cecropins) represent the major inducible defence against microbes, and their production is controlled by the same transcriptional elements as are used in the human immune system [4]. In mammals, including man, they (e.g., defensins) are the predominant protein species in the neutrophil, a dedicated cell designed for immediate response against invading organisms [5]. They are also found on the surface of the tongue, trachea, lungs and upper intestine and are thought to be a major antibacterial defence on mucosal surfaces [6].

Cationic antibacterial peptides appear to have evolved through convergent evolution [3]. Despite their similar high content of basic amino acids and common functional role, they have dramatically different three-dimensional structures which can be $\beta$-sheets, $\alpha$-helices, extended helices or loops, consistent with them having different origins $[3,6]$. More than 150 different cationic peptide structures have been de- scribed in the literature, and a single organism can have one or all of the above structural classes.

\section{Outer membranes as a drug barrier}

The outer membranes of gram-negative bacteria are selectively permeable to drugs [7-9]. Hydrophilic permeation occurs through the water-filled channels of proteins termed porins. Because the channels have limited sizes, only molecules smaller than a certain exclusion limit can pass through these channels, and as molecules (e.g., $\beta$-lactam antibiotics) approach this limiting size they are slowed down by intermolecular interactions with the amino acids lining the channel. As porin channels represent $<5 \%$ of the surface area of the Escherichia coli outer membrane, this means that the presence of the outer membrane reduces the potential rate of uptake of $\beta$-lactam antibiotics in $E$. coli by a factor of $>1000$. In Pseudomonas aeruginosa and other non-fermenters with high intrinsic resistance, antibiotic permeation rates across the outer membrane are further reduced by up to 100 -fold.

In addition to porin-mediated uptake of water soluble molecules, two extra uptake systems exist. Polycations, including aminoglycosides and polymyxins, use a selfpromoted uptake system. Hydrophobic drugs on the other hand cross directly through the outer-membrane bilayer, and studies with steroids suggest that penetration rates are $10-100$-fold lower than those observed for other kinds of biological membranes.

Restricted outer-membrane permeation cannot by itself explain intrinsic antibiotic resistance, because even at slow, limited permeation rates, equilibration of antibiotic concentrations across the outer membrane will occur in $0.2-100 \mathrm{~s}$, depending on the antibiotic in question. Rather, it must be combined with a secondary resistance mechanism that can take advantage of slow exposure to antibiotic; e.g., periplasmic $\beta$-lactamases or active efflux of antibiotic. Nevertheless, restricted outer-membrane permeability plays a critical role in the intrinsic antibiotic resistance of gram-negative bacteria as increasing or decreasing 
outer-membrane permeability results in antibiotic super-susceptibility or resistance respectively.

\section{Interaction of peptides with gram-negative bacteria}

Recent research has provided a rather complete picture of how cationic peptides interact with gram-negative bacteria and although this interaction is described here for indolicidin, a bovine neutrophil-derived peptide [10], similar data have been gathered for other types of peptides $[3,5]$. Indolicidin, like other polycations, crosses the outer membrane by self-promoted uptake. This involves the initial competitive displacement of divalent cations $\left(\mathrm{Mg}^{2+}\right.$ or $\left.\mathrm{Ca}^{2+}\right)$ from their binding sites on surface lipopolysaccharide (LPS). Because indolicidin is far larger than a divalent cation, it causes distortion of the outer-membrane structure. On contacting the cytoplasmic membrane, indolicidin inserts into this membrane under the influence of the transmembrane electrical potential gradient, which is oriented interior negative such that cationic indolicidin molecules will pass from the periplasm into the cytoplasmic membrane. A potential of at least $-80 \mathrm{mV}$ is required to promote such insertion and the result is association of indolicidin molecules to form channels in the cytoplasmic membrane, through which essential cellular solutes leak. Indolicidin forms rather defined channels, although other cationic peptides form channels of variable sizes.

This mechanistic picture explains many of the more desirable antibacterial features of cationic peptides. The ability to bind to LPS explains their antiendotoxic activity [11], as endotoxin is basically LPS. The proposed permeabilisation of the outer membranes to other cationic peptide molecules is consistent with the observed increased outer-membrane permeability to probe molecules, and the known synergy of certain cationic peptides with conventional antibiotics [12,13]. Also, the physical mode of action in forming channels explains the fact that mutations to resistance have not been observed with cationic peptides [6], as alteration of membrane structure to prevent insertion and channel formation is far more difficult than remodelling target enzymes.

\section{Design of novel peptides}

There is already considerable structural diversity among cationic peptides [3], and considerable aminoacid sequence heterogeneity exists within given structural classes. However, with a theoretical $20^{13}$ possible variants of a 13 amino-acid peptide, there is ample scope for designing improved peptides. Basically, two methods have been used: systematic variation in selected amino acids, and fusion of parts of individual peptides. New peptide design can be assisted by computer modelling because, as a general rule, active peptides should fold in three dimensions to form a structure with the charged and hydrophilic amino acids on one face, and the hydrophobic (membrane-interacting) amino acids on the other. Production can be carried out by chemical peptide synthesis or by recombinant DNA methods [14] that permit design of novel variants by molecular biological mutagenesis procedures. The ability to produce antibacterial peptides by molecular biological methods, makes them potentially the first class of recombinant antibiotics.

\section{Therapeutic potential}

Peptides have many exciting properties that would seem to recommend them as novel antibiotics. It is still too soon to state whether they will achieve their potential, although some clinical studies are underway. In animal studies of efficacy, protection has been observed against: Pseudomonas infections in neutropenic mice (with $\alpha$-helical peptides [10]) fungal infections (with liposomal indolicidin [15]); and Helicobacter infections (with nisin) in mice. In addition, the $\alpha$-helical peptide, magainin, has been shown to enhance activity of a $\beta$-lactam antibiotic, cafpirome [12], and to exhibit anti-endotoxin activity in galactosamine-sensitised mice [11]. Two cationic peptides are currently in clinical trials: MSI-78 against diabetic foot ulcer infections is currently in phase III trials; nisin (a natural product from Lactococcus) is in phase I clinical trials against Helicobacter gastric infections. Several other cationic peptides are under development. The next 5 years should reveal whether these peptides will really be the first new structural class of antibiotics in three decades.

Research on these subjects was funded by MRC, Canada, the Canadian Cystic Fibrosis Foundation, the Canadian Bacterial Diseases Network and Micrologix Biotech Inc.

R. E. W. HANCOCK, Department of Microbiology and Immunology, University of British Columbia, Vancouver, British Columbia, Canada V6T 1 Z3.

\section{References}

1. Neu HC. Infection problems for the 1990's - do we have an answer? Scand J Infect Dis Suppl 1993; 91: 7-13

2. Silver L, Bostian K. Screening of natural products for antimicrobial agents. Eur J Clin Microbiol Infect Dis 1990; 9: $455-461$.

3. Hancock REW, Falla T, Brown MH. Cationic antibacterial peptides. In: Poole RK (ed) Advances in microbial physiology. London, Academic Press. 1995: 135-75.

4. Engström Y, Kadalayil L, Sun SC, Samakovlis C, Hullmark D, Faye I. kappa B-like motifs regulate the induction of immune genes in Drosophila. J Mol Biol 1993; 232: 327-333.

5. Lehrer RI, Ganz T, Selsted ME. Natural peptide antibiotics from neutrophils. ASM News 1990; 56: 315-318.

6. Boman HG. Peptide antibiotics and their role in innate immunity. Annu Rev Immunol 1995; 13: 61-92.

7. Nikaido $H$, Vaara M. Molecular basis of bacterial outer 
membrane permeability Microbiol Rev 1985; 49: 1-32.

8. Hancock REW, Karunaratne DN, Bernegger-Egli C. Molecular organization and structural role of outer membrane macromolecules. In: Ghuysen J-M, Hakenbeck R (eds) Bacterial cell wall (New comprehensive biochemistry, vol. 27). Amsterdam, Elsevier Science Publishers. 1994: 263-279.

9. Hancock REW. The outer membrane as a drug barrier. Trends Microbiol Sci 1996 (in press).

10. Falla TJ, Karunaratne DN, Hancock REW. Mode of action of the antimicrobial peptide indolicidin. J Biol Chem 1996; 271 : 19298-19303.

11. Gough M, Kelly NM, Hancock REW. Anti-endotoxic potential of cationic peptide antimicrobials. Infect Immun 1996 (in press).
12. Darveau RP, Cunningham MD, Seachord CL et al. Beta-lactam antibiotics potentiate magainin 2 antimicrobial activity in vitro and in vivo. Antimicrob Agents Chemother 1991; 35: 11531159.

13. Vaara M, Porro M. Group of peptides that act synergistically with hydrophobic antibiotics against Gram-negative enteric bacteria. Antimicrob Agents Chemother 1996; 40: 1801-1805.

14. Piers KL, Brown MH, Hancock REW. Recombinant DNA procedures for producing small antimicrobial cationic peptides in bacteria. Gene 1993; 134: 7-13.

15. Ahmad I, Perkins WR, Lupan DN, Selsted ME, Janoff AS Liposomal entrapment of the neutrophil-derived peptide indolicin endows it with in vivo anti-fungal activity. Biochim Biophys Acta 1995; 1237: 109-114. 